\title{
Title: Identifying Biomarkers and Mechanisms of Toxic Metal Stress with Global Proteomics
}

Principal Investigator: Susan M. Miller, Ph.D., Department of Pharmaceutical Chemistry, University of California San Francisco, $60016^{\text {th }}$ St., UCSF MC 2280, San Francisco, CA 94158-2517; email: smiller@cgl.ucsf.edu

Final Report: 05/15/2007 - 05/14/2011

Recipient Organization: The Regents of the University of California, San Francisco

DOE Award No: DE-FG02-07ER64409, Project ID: 0013347:

Researchers working on project:

\begin{tabular}{|l|l|l|}
\hline Name & Position & Current position \\
\hline $\begin{array}{l}\text { Benjamin J. } \\
\text { Polacco }\end{array}$ & $\begin{array}{l}\text { Postdoctoral fellow, } \\
\text { Research Specialist }\end{array}$ & Research Specialist - UCSF \\
\hline Baoyu Hong & Postdoctoral fellow & QC manager - local company \\
\hline Angie Perez & Postdoctoral fellow & Toxicology consultant \\
\hline
\end{tabular}

This project is part of a collaborative grant funded under three separate awards. Other awardees include Anne O. Summers, Ph.D., Department of Microbiology, University of Georgia, DOE Award No. ER64408, Project ID 0013344, and Mary Lipton, Ph.D., PNNL, DOE Lab Project \#51667.

\section{Executive Summary}

$\mathrm{Hg}$ is a wide-spread contaminant in the environment and is toxic in all of its various forms. Data suggest that $\mathrm{RHg}^{+}$and $\mathrm{Hg}^{2+}$ are toxic in two ways. At low levels, $\mathrm{Hg}$ species appear to disrupt membrane-bound respiration causing a burst of reactive oxygen species (ROS) that further damage the cell. At higher $\mathrm{Hg}$ concentrations, $\mathrm{RHg}^{+}$and $\mathrm{Hg}^{2+}$ may form adducts with cysteine- and selenocysteine-containing proteins in all cellular compartments resulting in their inactivation. Although these mechansims for toxicity are generally accepted, the most sensitive targets associated with these mechanisms are not well understood. In this collaborative project involving three laboratories at three institutions, the overall goal was to develop of a mass spectrometry-based global proteomics methodology that could be used to identify Hg-adducted (and ideally, ROSdamaged) proteins in order to address these types of questions. The two objectives of this overall collaborative project were (1) to identify, quantify, and compare ROS- and $\mathrm{Hg}$-damaged proteins in cells treated with various $\mathrm{Hg}$ species and concentrations to test this model for two mechanisms of $\mathrm{Hg}$ toxicity, and (2) to define the cellular roles of the ubiquitous bacterial mercury resistance (mer) locus with regards to how the proteins of this pathway interact to protect other cell proteins from $\mathrm{Hg}$ damage. The specific objectives and accomplishments of the Miller lab in this project included: (1) Development of algorithms for analysis of the Hg-proteomic mass spectrometry data to identify mercury adducted peptides and other trends in the data. (2) Investigation of the 
role of mer operon proteins in scavenging $\mathrm{Hg}(\mathrm{II})$ from other mer pathway proteins as a means of protecting cellular proteins from damage.

\section{Synopsis of Results}

\section{(1) Development and analysis of computational filter to identify $\mathrm{Hg}$-adducted} peptides in data from global LC-MS/MS data - One of the primary goals of the Miller laboratory in this project was to develop the computational tools to analyze the mass spectrometry data for the presence of Hg-adducted peptides. The basis for the approach is the natural occurrence of seven stable isotopes of $\mathrm{Hg}$, six of which are present at $\geq$ $5 \%$ natural abundance. Furthermore, several of the heavier isotopes have higher abundances than those of the lighter isotopes, which is the opposite of the situation with the typical elements of peptides including $\mathrm{C}, \mathrm{N}, \mathrm{O}, \mathrm{P}$ and $\mathrm{S}$, where the lighter isotopes are the most abundant. As a result, the isotope distribution (or pattern) observed for a $\mathrm{Hg}$-peptide adduct is more symmetrical than that of a simple peptide allowing identification of the Hg-adducts in high resolution mass spectral data. A manuscript describing the algorithm along with a thorough statistical analysis of its utility for identifying different types of $\mathrm{Hg}$-adducted peptides from both pure protein preparations and from global proteomic preparations was published in 2011 (Polacco et al - see below). Dr. Polacco is continuing extensive analyses of the proteomics data as the collaborative group is preparing one or more additional manuscripts describing the results of the work and their biological and structural implications.

(1a) Experimental controls for proteomics procedures - Although $\mathrm{Hg}(\mathrm{II})$ - and $\mathrm{RHg}$ adducts of proteins had been observed by our groups and by other laboratories, this project was the first to attempt to examine these adducts in whole proteomes. Thus we needed to devise appropriate methods to retain these adducts throughout the experimental steps of cell lysis, proteolysis of the lysates, chromatography prior to and inline with the mass spectrometry, as well as within the mass spectrometer itself. The Miller laboratory prepared a number of phenylmercury- and $\mathrm{Hg}(\mathrm{II})$-adducts of pure peptides and proteins that were then subjected to various steps in the procedure to define and optimize the experimental protocols and instrument settings to obtain the best recovery of $\mathrm{Hg}$-adducts. Further details of these controls, where appropriate will be included in the manuscripts mentioned above.

(2) $\mathrm{Hg}$ (II) transfer from MerB to MerA - The other goal of the Miller laboratory was to evaluate the ability of proteins from the mer operon to recover $\mathrm{Hg}(\mathrm{II})$ that had leaked into the cell and become bound to other cellular proteins. As we had previously shown that the NmerA domain of a $\gamma$-proteobacterial MerA could abstract $\mathrm{Hg}(\mathrm{II})$ from the cellular protein thioredoxin, we anticipated a role for this domain in acquiring $\mathrm{Hg}(\mathrm{II})$ from both mer pathway proteins and other cellular proteins, and focused our attention on interactions of this domain (expressed as a separate protein) with other proteins. As the mass spectrometry aspects of the project was at the stage of methods development at the outset of this award, we decided to focus our attention initially on interactions of NmerA with other mer pathway proteins as no thorough investigations of interactions of this type had previously been undertaken. Previous steady-state type studies of an 
organomercurial lyase (MerB) from a $\gamma$-proteobacterial mer operon qualitatively suggested that $\mathrm{Hg}(\mathrm{II})$ could be transferred directly between MerB and MerA. However, the use of low levels of one or both proteins in those studies and the inclusion of a small molecule thiol in the reactions obscured quantitative comparison of the possible pathways for the transfer. Based on analysis of the MerB crystal structure we developed a spectroscopic probe to monitor the kinetics of $\mathrm{Hg}$ (II) removal from MerB and studied the reaction using stopped flow spectroscopy. These studies provided important insights into the relative efficiency of direct $\mathrm{Hg}(\mathrm{II})$ transfer between the proteins compared with transfer mediated by the cellular thiol buffer, insights into which protein domains and specific residues are involved in direct transfer of $\mathrm{Hg}$ (II) between the proteins, and insights into possible alternative mechanisms in other pathways. The first manuscript on this work is published (B. Hong et al - see below) and two more manuscripts that describe the role of key amino acid residues in MerB will be submitted in the near future. In addition, results from these studies were presented in three seminars and one poster (as well as in posters at the SBR PI meetings). As this line of investigation proved quite interesting and results from the proteomics investigation came more slowly, we had to delay investigation of interactions of NmerA with cellular proteins but still plan to pursue that in the future.

\section{Posters, Seminars, Publications}

\section{Posters}

Baoyu Hong, Rachel Nauss, Susan M. Miller. Direct Transfer of Mercuric lons from MerB to MerA: Co-evolved Enzymes from a Mercury Detoxification Pathway. Poster at American Chemical Society, April 2010

Ben Polacco, SO Purvine, EM Zink, SP LaVoie, MS Lipton, AO Summers, SM Miller. Discovering $\mathrm{Hg}$ and Se peptides in whole proteomes using natural isotope distributions in LC-MS/MS. Poster at Tenth International Symposium on Mass Spectrometry in the Health and Life Sciences: Molecular and Cellular Proteomics, August 2011

\section{Seminars}

Biomolecular Mechanisms of Mercury Transfers and Transformations by Proteins of the mer Operon. Susan M. Miller, seminar at American Geophysical Union Meeting, Dec 2008

Bacteria Facing the $\mathrm{Hg}$ Challenge - Evolutionary Conserved and Divergent Features of Mercury Detoxification Enzymes. Susan M. Miller, seminar at Trends In Enzymology, June 2010

Structural Control of $\mathrm{Hg}(\mathrm{II})$ Transfer Between Enzymes of Mercury Detoxification. Susan M. Miller, seminar at Enzymes, Coenzymes and Metabolic Pathways Zing Conference, Nov 2011. 


\section{Published Papers acknowledging DE-FG02-07ER64409 thus far}

Baoyu Hong, Rachel Nauss, lan M. Harwood, \& Susan M. Miller. Direct Measurement of $\mathrm{Hg}$ (II) Removal from Organomercurial Lyase (MerB) by Tryptophan Fluorescence: NmerA Domain of Coevolved $\gamma$-Proteobacterial Mercuric Ion Reductase (MerA) Is More Efficient than MerA Catalytic Core or Glutathione. (2010) Biochemistry 49, 8187-8196.

Benjamin J. Polacco, Samuel O. Purvine, Erika M. Zink, Stephen P. LaVoie, Mary S. Lipton, Anne O. Summers \& Susan M. Miller. Discovering Mercury Protein Modifications in Whole Proteomes Using Natural Isotope Distributions Observed in Liquid Chromatography-Tandem Mass Spectrometry. (2011) Mol. Cell Proteomics 10.8, M110.004853-1-13

See A.O. Summers award DE-FG02-07ER64408 for additional presentations on the overall project. 